\title{
The worldwide trend of using botanical drugs and strategies for developing global drugs
}

\author{
Kyungseop Ahn ${ }^{*}$ \\ Natural Medicine Research Center, Korea Research Institute of Bioscience and Biotechnology, Cheongju 28116, Korea
}

\begin{abstract}
Natural product drugs, or botanical drugs, are drugs composed of natural substances which have constituents with healthenhancing or medicinal activities. In Korea, government-led projects brought attention to botanical drugs invigorating domestic botanical drug industry. Foreign markets, as well, are growing bigger as the significance of botanical drugs stood out. To follow along with the tendency, Korea puts a lot of effort on developing botanical drugs suitable for global market. However, standards for approving drug sales vary by countries. And also, thorough standardization, certification, clinical studies and data of these will be required as well as data confirming safety and effectiveness. Meanwhile, as an international exchange in botanical drug market continues, the importance of plant resources was emphasized. Thus countries' ownership of domestic natural resources became vital. Not only establishing a systematic method to secure domestic plant resources, but also cooperation with other countries on sharing natural resources is essential to procure natural resources effectively. Korea started to show visible results with botanical drugs, and asthma/COPD treatment made out of speedwell is one example. Sufficient investment and government's active support for basic infrastructure for global botanical drugs will bring Korea to much higher level of botanical drug development. [BMB Reports 2017; 50(3): 111-116]
\end{abstract}

\section{INTRODUCTION}

Natural products, in a broad sense, is a generic term for plants, animals, minerals, microorganisms and their metabolites found in nature.

In Korea, the term 'natural product drug' was definedaccording to the enactment of 'Natural Product Drug R\&D Acceleration Law' in 2000- as drugs researched and developed

*Corresponding author. Tel: +82-43-240-6113; Fax: +82-43-2406129; E-mail: ksahn@kribb.re.kr

https://doi.org/10.5483/BMBRep.2017.50.3.221

Received 20 December 2016

Keywords: Botanical drug, IND, Natural product, Plant extract bank, Reverse pharmacology using substances from natural products that contain new constituents and efficacies.

The U.S. coined the official term 'botanical drug' for natural products, referring to drugs manufactured with plant substances, including algae, microfungi (the term botanical drugs will be used for natural product drugs hereafter). While many vendors promote the use of botanical products as dietary supplements, only a select number of products have been approved by the U.S. Food and Drug Administration (FDA) for use as prescription drugs. The first botanical drug approved by the FDA was Veregen ${ }^{\circledR}$, a treatment for genital and perianal warts that is derived from green tea (Camellia sinensis Kuntze) (1). A number of years later, the FDA approved another New Drug Application (NDA) for the drug Fulyzaq ${ }^{\mathrm{TM}}$, an indicator drug for HIV-associated diarrhea extracted from the blood-red latex of the South American croton tree (Croton lechlerii Müll. Arg) $(2,3)$.

In Europe, they use the term herbal medicinal product (HMP) to encompass all drugs that contain one of more kind of herbal substances in the herbal preparation. The Chinese developed TCM (Traditional Chinese medicine), a prescription of their traditional medicines, based on their own experiences of its usage, and eastern ideas.

\section{DEVELOPMENT TRENDS OF BOTANICAL DRUGS}

In 2000, 'Natural Product Drug R\&D Acceleration Law' was established as institutional support from the Korean government and, based on this law, the government and various institutes initiated a 5-year-plan of research and development of botanical drugs. Botanical drugs drew more interest from the public when botanical drugs were selected as one of the five tasks of a government-derived project. In the first half of 2011 alone, three products, including the Shinbaro Capsule from Greencross, Synatura Syrup from Ahn-gook, Motilitone from Dong-A were approved for marketing, and in 2012, the PMG Pharm's osteoarthritis treatment and Layla tablet, Yungjin's atopy cure, Utoma, was accepted as a botanical drug. In 2013, according to the data from UBIST, a program aggregating statistics on drugs, the Stillen tablet from Donga-ST raised more than 60 million dollars, Ahn-gook raised 30 million dollars with Synatura Syrup, 18 million dollars from Motilitone, and 36.4 million dollars from Joins Tablet, 6.7

ISSN: 1976-670X (electronic edition)

Copyright (C) 2017 by the The Korean Society for Biochemistry and Molecular Biology

(c) This is an open-access article distributed under the terms of the Creative Commons Attribution Non-Commercial License (http://creativecommons.org/licenses/by-nc/4.0) which permits unrestricted non-commercial use, distribution, and reproduction in any medium, provided the original work is properly cited. 
million dollars from SK Chemical, 5.2 million dollars from Shinbaro Capsule, and 5.2 million dollars from Layla Tablet (5.2 million as prescription performance). These performances indicate a successful settlement of botanical drugs in the domestic market. Encouraged by these facts, pharmaceutical companies reinforced their developing processes of botanical drugs. Currently, 24 companies are researching and developing botanical drugs, and $23 \%$ of the investigational drugs in the pipeline are botanical drugs. These companies mainly focus on digestive system, metabolic and central nervous system diseases (4).

Meanwhile, the size of the global market, including medical supplies, functional foods for health etc. is estimated to be 1 trillion dollars and is growing annually by 8 to 10 percent. About half of the medical supplies currently on sale are botanical drugs, or extracts composed of single-element substances from natural products. Tamiflu (Oseltamivir) is one typical example. Tamiflu was discovered from a natural substance called star anise which is a Chinese native plant, and its sales from all over the world reached 3 billion dollars. Besides Tamiflu, Aspirin, Taxol, Ginkgo Biloba extract, and plantain extract were developed and put on sale, raising big profits. In 2004, FDA established an industrial guideline for botanical drugs (Botanical Drug Guidance), as an effort to take the initiative in the botanical drug market. As a result, the FDA approved 868 kinds of Semisynthetic substances that originated as natural products since 1982 and developed 20 kinds of an anticancer drug derived from natural substances until 2002 (Science Times, 2010. 8). Veregen Ointment 15\% $\left(\right.$ Veregen ${ }^{\mathbb{R}}$ Phynova, warts treatment on pubic area, approved in 2006) made by Medigen, German Pharmaceutical, is one of the products that was approved by the FDA. This drug reached 4.5 million dollars in sales. Fulyzaq, the second botanical drug developed, is the first oral medication developed by Salix Pharmaceuticals using a proanthocyanidin polymer extracted from wild plants in the Amazon river basin, and this drug was approved for sales in 2012, as an orphan drug which relieves symptoms of diarrhea from AIDS patients. GW Pharmaceuticals, an English company, developed Sativex (Oromucosal Spray), a marijuana extract efficacious for rigidity due to multiple sclerosis. GW Pharm raised 11 million dollars with Sativex. China intends to found the world's biggest Chinese medication database. Sales of Chinese medication are $22.7 \%$ of the entire drug market and it is expected to grow at the same pace as the recorded high growth at an average of $35 \%$ annually over the past 5 years $(5,6)$.

\section{CHALLENGES IN DEVELOPING GLOBAL BOTANICAL DRUGS}

Although the global market of botanical drugs is expanding continuously, Korea's performance overseas is not quite satisfactory. Stillen, one of the representative botanical drugs in Korea, raised only 200 thousand dollars abroad after its release. Other domestically developed botanical drugs are no different when it comes to sales performance. Performances until now contrasts with the government's prediction that botanical drugs will be one of the major sources of income in Korea.

\section{Botanical drug approvals in the U.S.}

There have been numerous attempts to bring botanical drugs to the market through FDA approval, including $500+$ pre-IND meetings and IND applications, with limited success in reaching the final NDA stage. In fact, only two NDAs have been approved by the FDA so far (see Fig. 1). The authors suggest in this section three of the most commonly encountered complications that account for the low number of botanical drug NDAs submitted to the FDA for review $(7,8)$.

Insufficient Evidence for Efficacy: The FDA requires "adequate and well-controlled" multicenter clinical studies on any new drug candidate to document and support its safety and efficacy, and imposes the maximum level of scrutiny prior to approval. It is crucial for these efficacy studies to have a well-defined target population (according to the FDA protocol eligibility criteria), proper experimental controls (such as placebo or active treatment), appropriate outcome measures (agreed upon by the FDA), independent monitoring, and accurate analysis. Consequently, the single most common reason that any new drug candidate, including botanical drugs, fails to reach the NDA step is the failure to present statistically significant evidence for having efficacies of clinical relevance.

Unrealistic Goals and Expectations: Unrealistic expectations are often set forth by drug sponsors with insufficient experience with a drug development program, for multiple reasons, and the resulting miscommunication could hinder the drug approval process. For one, the initial stages of IND

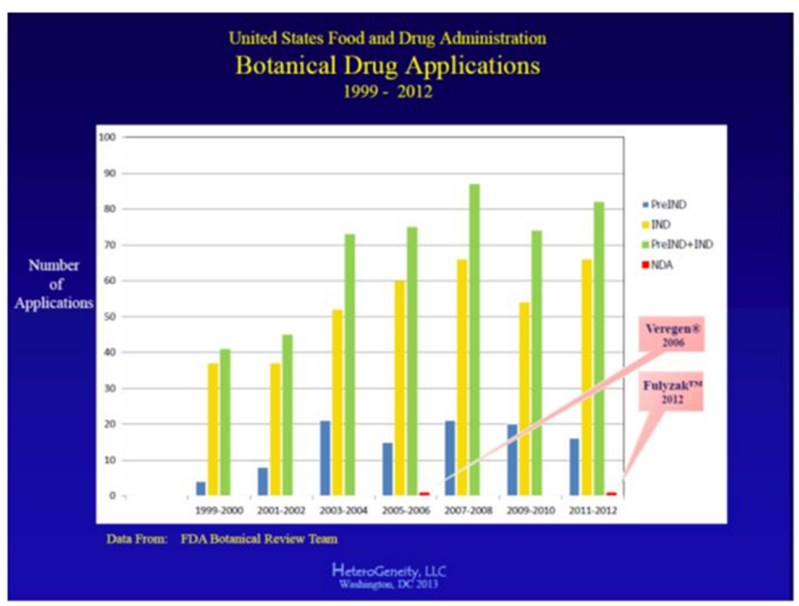

Fig. 1. Botanical drug applications in US FDA during 1999-2012. 
development is relatively less strict, and this may falsely suggest to new drug sponsors that botanical drugs, compared to conventional synthetic drugs, are less rigorously evaluated by the FDA. Another reason for drug sponsors to have unrealistic expectations is the confusion that arises from the lack of internationally standardized regulatory requirements for botanicals. In other words, many non-U.S. botanical drug sponsors, especially those that have no experience in fielding a drug development operation in the U.S., are not aware of the practical differences in regulations between the U.S. and a foreign market. As an anecdote, some sponsors do not reconcile the fact that "raw data" (chemistry, nonclinical safety testing, or clinical study databases), instead of data summaries or "expert" opinion, is required by U.S. regulations.

Insufficient Funding: It is possible that the development of botanical drugs in the IND stage suffers the loss of some or all project funding, which brings the project to a standstill. Several factors contribute to funding cuts: general economic climate; skepticism of investors; lack of patents (even if other forms of intellectual property are filed and secured); and/or inadequate project planning.

\section{STRATEGIES FOR DEVELOPING 'GLOBAL BOTANICAL DRUGS.'}

\section{Development of novel botanical drugs using traditional medicinal plant sources}

Botanical drugs are, by nature, plant-derivative materials and their complexes. This makes them unfit for conventional "single-target/single-drug" development processes and thus have been largely disregarded in the field of medicine. However, it is widely understood in synthetic medicine that the single-drug "magic bullet" strategy is not adequate for treating chronic illnesses (e.g. cancers, immune disorders, mental illnesses, cardiovascular diseases, lifestyle diseases) due to their complex pathogenetic mechanisms, and that a "multi-target/multi-component" approach involving control over a number of target sites is more effective (9-11).

Traditional herbal medicine, itself being a mixture of various components, corresponds to the "multi-target/multi-component" approach, with therapeutic effects that are clinically confirmedalbeit with no analytically defined mechanisms--through experience and knowledge accrued over a long history of treatment of chronic illnesses. The strategy for developing novel botanical drugs by reverse-engineering of traditional herbal medicine is called reverse pharmacology (Fig. 2). Reverse pharmacology involves the study of active ingredients based on traditional herbal medicine and formulations as well as the subsequent development of drug candidates or formulations for preclinical and clinical research. This is deemed to be the most optimal strategy for the development of cures for chronic illnesses because it utilizes materials (traditional herbs) with proven safety and clinical efficacy, which allows the development process to be opposite that of

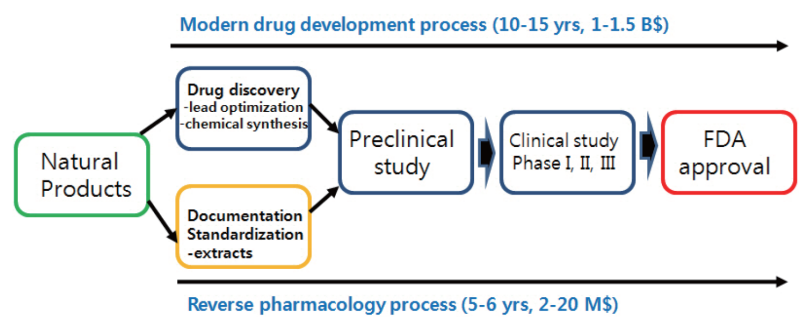

Fig. 2. Reverse pharmacology.

the initial stages of synthetic drug development and therefore reduces the cost and duration of development compared to conventional synthetic drug development methods $(12,13)$. Using knowledge from traditional Korean medicine in botanical drug development is also a reverse pharmacology approach with many successes reported worldwide, which prompted the reevaluation of traditional medicinal herbs and greatly spurred the research and development of botanical drugs.

\section{Keeping pace with global standards of botanical drug development}

For Korean botanical drugs to successfully compete in global market, there are some conditions institutes and pharmaceuticals to keep in mind. Since the licensing procedure of new drugs varies by countries, it is reasonable to begin development procedure in accordance with such country's distinct regulations.

Secondly, certification and standardization of botanical drugs, complex clinical studies, scientific data for sale approval are essential. For materials, specific origin of the medicine and securing bioequivalence is the most important and procuring bioequivalence is the most challenging part of drug development process. Equivalent production of the material is also a vital portion, scientification of plants, ecological environment, a record of cultivation should be conducted. If a company imports natural products as materials, they need to secure a reliable supply chain in terms of collection, cultivation, and importation for the seamless supply of medicine.

The third requisite is safety and effectiveness. All countries, including the US, Europe, and China, set safety as the top priority. They accept oriental medicine texts of cases of drug usage abroad to some extent, but mainly, they request data on drug-drug interaction (DDI), mechanism of action (MOA) and pharmacokinetic (PK) $(14,15)$.

Meanwhile, the Nagoya Protocol came into effect. There are articles related to ABS, agreement of access to genetic resources and benefit-sharing, in Nagoya Protocol which consequently acknowledges each country's ownership of its domestic natural resources. Therefore, biotechnology institutes and universities, especially those that are in the beginning 
process of developing botanical drugs, should be well informed about ABS articles. To sensibly deal with this issue, institutes should build a partnership with countries providing resources or such organizations. Also, they need to analyze regulation regarding access and benefit share in different countries and come up with counterplan for anticipated future problems. For counterplay, financial benefit sharing, technology transfer, co-ownership of patent and intellectual property may be possible (16).

\section{Promoting infrastructure on securing plant resources}

Industrial value of plant resource in drug market is prominent. Traditional plant resources were not only the key material for drug development, but also a core resource in the market for botanical drug products since $85 \%$ of the world's traditional medication derive from plants. Korean, China, Japan, India, Germany, countries that have long developed traditional medicine and herbal medication, are striving to procure plant resources countrywide to develop botanical drugs using plant extract. Specific strategies among countries might differ, but the point is they all acknowledge the significance of plant resource.

Korea has constructed their own method of securing plant resources. Korean Research Institute of Bioscience \& Biotechnology (KRIBB) founded South Korea's first massdistribution system named Korea Plant Extract Bank. Extract Bank provides researchers screening samples at the beginning of food and drug development phase, assisting researchers to acquire resources regardless of season, and holds vouchers of materials to provide the origin of the plantation, and subsidiaries preventing overexploitation of rare species. From 2000 to 2010, extract bank gathered 1,699 species of Korean native plants, which takes up $40 \%$ of entire Korean plant species, excluding garden plants and food crops, and made extracts with 5,164 samples separated by parts then offered them to researchers (total $416,829 / 2013.12$ ) as a part of supporting research of domestic natural substances (Fig. 3).

Identification process of Plant origin in PEB

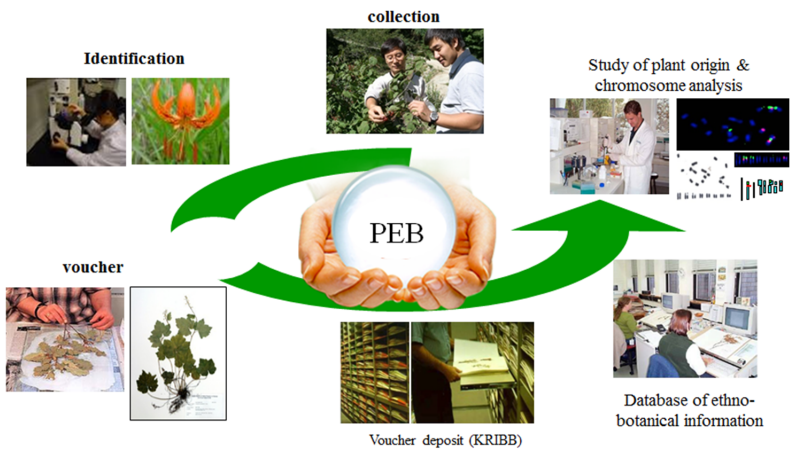

Fig. 3. Construction and use of the plant extract bank.
Meanwhile from 2006, in terms of extending a variety of resources, extract bank has launched 'International Biological Material Development Project' (2006-2016) and established 4 local centers near tropical/subtropics area (China, Indonesia, Costa Rica, Vietnam) where a broad range of life exists. Each year, through a formal agreement, extract bank assembles foreign resources $(27,000$ pieces of extract, 2015. 12) and establishes a database of basal activity(anti-inflammatory, cytotoxic, antioxidation, insecticide). After that, they give out foreign resources $(1,600,000$ pieces 2015 . 09) to researchers and provide them information based on the database (17).

\section{ENDEAVORS OF KOREAN PHARMACEUTICALS FOR GLOBALIZING DOMESTIC BOTANICAL DRUGS}

In spite of some challenges Korea had in globalizing domestic botanical drug products, some companies are starting to show visible results throughout the US and Europe. Korean government suggested 'Global Leading Natural Pharmaceutical Project' as a project to forge developing industries to the highest level and make internationally recognized drugs. With Donga-ST in charge, 10 pharmaceutical companies participated with the support of 16 industry-academic collaboration institutes. As a result, Donga-ST's new diabetic Neurotherapy treatment was approved by the US for clinical study phase I IND in April 2013, and recently got down to clinical trial. Motilitone is on standby for FDA's approval on phase II within the first half of 2014. COPD/asthma treatment from Yungjin Pharmaceutical completed phase I and is in the middle of phase II base on approval in 2014. For pharmaceuticals aiming Europe market, Greencross HS developed anticancer supplements and is in the process of phase I trials and SK Chemicals has set a goal of their asthma treatment to acquire authorization of Phase I trials in Europe (18).

All of this medicine were developed is Korea and later got approval at clinical studies from institutes of Europe and the US, quantitatively/qualitatively comparable with botanical drugs made in developed countries.

The KRIBB Natural Medicine Research Center developed asthma/COPD treatment, and the natural substance they used is speedwell genus (Pseudolysimachion genus) plants. Speedwell, according to Chinese medicine dictionary, is a kind of herb medicine used to treat loosen phlegm, chronic cough, and asthma/COPD. However, in Korea, there was no record of speedwells in 10 major books being utilized as herbal medicines, which means that the usage of speedwell was not extensively known to the traditional Korean medicine world. From the beginning of research, relying on the clinical effects referred to in old books, research center conducted clinical studies on animals, and they discovered active materials substantially effectively. Since 2006, these results were applied to patents in 12 countries. For the following study, selected mountain spike speedwells in which the aerial part is relatively larger has active components. Then, after 
identifying excellence for cultivation at the central inland area, they massively cultivated wild seeds proving phytoequivalence between wild type and cultivated type seeds.

Afterward, they transferred such results, along with the patent to Yungjin Pharmaceuticals in May 2010, and July of the same year, KRIBB Natural Medicine Research Center was selected as supporting organization of pharmaceuticals on 'Global Botanical Drug Development Project.' For the past 3 years, Natural Medicine Research Center conducted studies on standardization of raw material medicine/herb medicine suitable for US guideline of clinical studies, and biological researchers on animal effectiveness/biomarker/MOA. Ultimately, KRIBB successfully supported Yungjin Pharmaceutical to complete phase I trials in the US.

\section{CONCLUSION}

The International perspective of drug development recently has been rapidly changing. As the world's population is constantly aging, and lifestyle diseases and chronic diseases have become more prevalent, and more and more people pursue improved quality of life which focuses on preventing diseases, drug development shifted its focus towards treating aging, cancer, chronic diseases, and lifestyle related diseases. Consequently, traditional medication, which safety and clinical effects had been confirmed over a long period of time for treating diseases mentioned earlier, and botanical drugs which stand on the basis of traditional medication are expected to grow tremendously. However, Korea relies most of its resources on foreign countries, and it can be a hindrance in developing botanical drugs. To resolve this matter, externally, Korea needs to establish a systematic network with countries possessing such resources and internally, needs to build up well-organized infrastructure on procuring natural resources residing in Korea. Lately, global trend has been towards acknowledging country's claim on its biological resources, and in the close future, countries providing biological resources (including intellectual resources) may demand their share of profits produced by developing natural resources. This ongoing trend would be a serious limitation to Korea's development strategy with foreign resources, so Korea needs to prepare plans to resolve this matter. In addition, basic structures for the legal system, analyzing and standardizing system for improving quality and GAP cultivation management of domestic natural resources should be set up, and continuously supervised. Government's active support and companies' investment based on this structure will bring forward the days that Korea moves up to a higher level on developing botanical drugs.

\section{ACKNOWLEDGEMENTS}

This study was supported by grants from the Korea Research Institute of Bioscience and Biotechnology Research Initiative Program (KGM 1221622) of the Republic of Korea.

\section{CONFLICTS OF INTEREST}

The authors have no conflicting financial interests.

\section{REFERENCES}

1. Blumenthal M (2006) FDA approves special green tea extract as a new topical drug for genital warts. HerbalGram (Austin, Tex.) November 9

2. Liscinsky M (2012) FDA approves the first anti-diarrheal drug for HIV/AIDS patients. US Food and Drug Administration (Silver Spring, MD) December 31

3. American Botanical Council (2013) FDA approves CRO Elem eras first-several botanical drug. American Botanical Council (Austin, TX) January 2

4. Biotech Policy Research Center (2012) White paper on Research and Development in the Korean Pharmaceutical Industry. Biotech Policy Research Center 2012

5. Report on the Bioproducts Market and Trends in Biotechnology Development (2010) Ministry of Knowledge Economy/Korea Evaluation Institute of Industrial Technology/Korea Biotechnology Industry Organization

6. Biotech Policy Research Center (2012) Trends in Research and Development on Novel Botanical Medicine. Biotech Policy Research Center

7. US Food and Drug Administration (2013) Investigational New Drug application. US Food and Drug Administration September

8. US Food and Drug Administration (2013) New Drug Application. US Food and Drug Administration website April 30

9. Morphy R and Rankovic Z (2009) Designing multiple legends-medicinal chemistry strategies and challenges. Curr Pharm Des 15, 587-600

10. Zimmermann GR, Lehar J and Keith CT (2007) Multitarget therapeutics: when the whole is greater than the sum of the parts. Drug Discov Today 12, 34-42

11. Frantz S (2005) Drug discovery: playing dirty. Nature 437, 942-943

12. Parwardhan B and Mashelkar RA (2009) Traditional medicine-inspired approaches to drug discovery: can Ayurveda show the way forward? Drug Discov Today 14, 804-811

13. Patwardhan B, Vaidya ADB, Chorghade $M$ and Joshi SP (2008) Reverse pharmacology and systems approaches for drug discovery and development, Curr Bioact Compd 4, $1-11$

14. Korea Biotechnology Industry Organization (2014) Seminar Presentation on the Strategy for Global Clinical Expansion of New Drug Development, Korea Biotechnology Industry Organization

15. Quality Standardization Based Botanical Drug Development Center (2013) Symposium Presentation on the Botanical Drug Material Development and Overseas Expansion Strategies. Quality Standardization Based Botanical Drug Development Center

16. Lee K (2014) Countermeasures by the Bio- and Pharma- 
ceutical Industries against the Implementation of the Nagoya Protocol Regarding Access and Benefit-sharing. Gyeonggi Bio Insight

17. Oh S (2011) Status of Botanical Medicine Based on
Plant-derivative Materials. Bioin Special

18. Son M (2014) Strategies and Case Studies of Research and Development of Novel Botanical Medicine by DongaST. Gyeonggi Bio Insight 